\title{
Otoacoustic emissions
}

Otoacoustic emissions (OAEs) are sounds produced by the healthy inner ear. To many people this is a surprising statement. After all, as far as we know, the eye does not emit light; so why should the ear produce sound? The answer lies in an understanding of current theories of cochlear function.

Most of us are familiar with the travelling wave theory which was developed by Georg von Békésy in the 1940s. ${ }^{1}$ Von Békésy studied the effect of sound upon the excised human cochlea. He discovered that the action of the stapes set up a wave in the basilar membrane that progressed from base to apex. When the frequency of the stimulus was altered, the travelling wave would peak at different sites on the membrane. High frequencies caused maximal vibration at the base of the cochlea whereas lower frequencies had a greater effect near the apex. In fact, each site on the basilar membrane had its own characteristic frequency that always produced maximum resonance at that point. Von Békésy proposed that the peak of the travelling wave was detected by the local hair cells, which responded by depolarising and initiating a sensory nerve signal. In this way, the ear was able to distinguish between sounds of different frequency.

The travelling wave theory suggests that the cochlea is entirely passive. Over the last two decades, however, it has been modified because experiments on live cochleas have produced findings which imply an additional, active process. For instance, the displacement of the basilar membrane is far greater at its characteristic frequency than the theory would predict. ${ }^{2}$ Also, as David Kemp found in 1978 , sounds can be recorded in the external ear that are almost certainly of cochlear origin. ${ }^{3}$ On occasion these emissions contain more energy than the eliciting stimulus. ${ }^{4}$ These findings led to the theory of a 'cochlear amplifier'. 5

The exact nature of the cochlear amplifier is unknown but it almost certainly involves the mechanical action of the outer hair cells upon the basilar membrane. ${ }^{6}$ In any event, it is generally agreed that some active mechanism causes the travelling wave to reach a very abrupt peak at, or near, its site of characteristic frequency. It is the sharpness of the peak which accounts for the exquisite sensitivity of the mammalian ear. As a by-product, the amplifier produces a secondary disturbance of the basilar membrane. This generates another, lower amplitude wave which passes back along the membrane and through the middle ear (see figure). It is this wave which emerges in the external ear as an OAE. ${ }^{7}$

The potential of OAEs as a hearing test was apparent to Kemp from the results of his earliest experiments. ${ }^{3}$ Kemp found that OAEs could be recorded from normal adult ears but not from those with hearing impairments. Subsequent studies on individuals of all ages have confirmed that normal ears almost always produce emissions while those with a hearing threshold of over $30 \mathrm{~dB}$ do not. ${ }^{8}$ The second great potential of OAEs is their ease of measurement. All that is required is an aural probe containing a sensitive microphone and a loudspeaker to deliver the stimulus. The minute acoustic responses that contain the OAEs are then summated and displayed by a personal computer program. Technicians can be trained to use the equipment in a couple of days.

Following Kemp's discovery, several other types of OAEs have been identified. ${ }^{78}$ These include spontaneous OAEs which, especially in young children, may occasionally be loud enough for other people to hear. ${ }^{8}$ In clinical practice, however, most researchers have used Kemp's original emissions which are now usually known as transient evoked OAEs. As this is particularly true of studies in childhood, the remainder of this article will concentrate on the use of transient evoked emissions.

\section{Otoacoustic emissions as a screening test}

Transient evoked emissions are essentially an all-ornothing response. Normal ears produce OAEs, whereas ears with hearing losses of any degree simply fail to produce a response. ${ }^{9}$ Furthermore, the loudness of the stimulus required to elicit an emission bears little resemblance to the subject's auditory threshold. ${ }^{89}$ For these reasons transient emissions are of little use in determining the severity of any hearing loss. However, because of their all-or-nothing response and the simplicity of their detection, OAEs do have great potential as a screening test.

Certain groups of newborn infants, such as those who are admitted to newborn intensive care units, are at risk of sensorineural hearing loss. Therefore, this population is often routinely screened for hearing impairment. Several techniques, such as the auditory response cradle, have been developed for this purpose. However, before the introduction of practical OAE machines, auditory brainstem responses (ABR) were the only objective test that was sufficiently widely available. This electrophysiological technique is of proved efficacy, ${ }^{10}$ but it is time consuming and technically demanding. ${ }^{11}$ Also, as electrodes are attached to the scalp, ABR is often perceived as being more invasive than OAE recording.

Several research groups have used transient evoked OAEs for high risk neonatal screening. Notably, independent studies in Sheffield and Southampton have compared OAEs with ABR in a total of over one thousand infants. ${ }^{12}{ }^{13}$ Both groups found that OAEs can be recorded in half the time of $\mathrm{ABRs}$ and are generally very well tolerated. Compared with hearing status at 8 to 18 months, both OAEs and ABR had a sensitivity of $100 \%$ for sensorineural hearing loss. OAEs had a specificity of around $80 \%$ whereas the figure for ABR was about $90 \%$. OAE screening therefore failed more normal ears. Nevertheless, the Sheffield group concluded that OAE screening is still worthwhile because of its simplicity. ${ }^{12}$ They suggested that OAEs could be used as an initial screen with the more complicated ABR being reserved for those who fail. The Southampton investigators reached similar conclusions. ${ }^{13}$ They calculated that the two tier screen would have a specificity of over $99 \%$. The encouraging results of these studies have led to the introduction of OAE screening into many neonatal units.

Unfortunately, OAE screening may not always be so effective. In recent years, the high risk neonatal programme in one unit has had a false positive rate of about $50 \%{ }^{14}$ The low specificity seems to be caused by difficulties in interpretation and technical problems peculiar to very low birthweight infants.

In view of these contrasting reports on the efficacy of OAEs, it is clear that data are needed from a much larger number of subjects. The Institute of Hearing Research in Nottingham is currently leading a multicentre study which has already screened thousands of high risk infants. A European database is also being constructed. Hopefully, these two initiatives will be able to reach firm conclusions regarding the sensitivity and specificity of high risk OAE screening. 


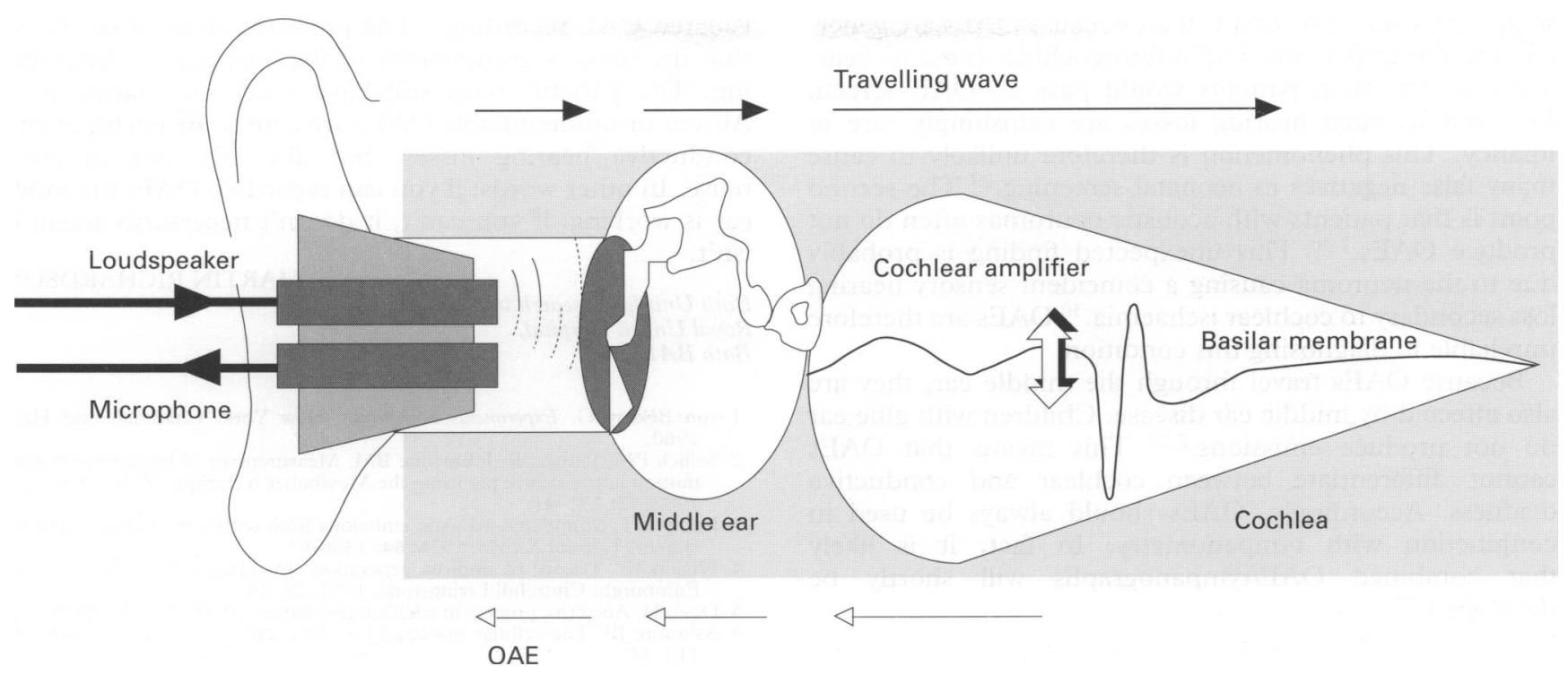

Production of an $O A E$.

Most infants with congenital sensorineural hearing loss do not fall into a high risk category. ${ }^{15}$ These children are sometimes not identified until they have reached the age of 2 or 3 years. ${ }^{1617}$ As there is also some evidence that early intervention improves language skills in this population, ${ }^{18}$ there is a case for routine screening of all newborn children. Evoked emissions may be particularly useful for this purpose and several trials of universal OAE screening have been started. The largest such study is in the American state of Rhode Island, where all newborn infants have been screened since $1990 .{ }^{19}$ Initial reports suggest that the programme will detect all babies with sensorineural deafness. Specificity is about $80 \%$ when compared with hearing status at 6 months of age.

Although universal OAE screening is still experimental, the Rhode Island study has already influenced health policy in the USA. In 1993 the National Institutes of Health declared that all American children should undergo screening for hearing impairment before the age of 3 months. ${ }^{20}$ The preferred method is neonatal OAE followed by ABR in those who fail.

The wisdom of this approach has been challenged, however, ${ }^{21}$ and many questions remain about the feasibility and cost effectiveness of universal screening. For instance, given that the incidence of congenital sensorineural hearing loss is about $0 \cdot 1 \%, 17$ an OAE screen with a specificity of $80 \%$ will fail 200 normal babies for every one with hearing impairment. Moreover, because babies who pass the Rhode Island screen are not followed up, the long term sensitivity of the test cannot be ascertained. The optimal timing of the test is also problematic. Clearly, the most practical time is while the child is on the postnatal ward. To achieve maximum coverage the test should therefore be performed during the first few days of life. However, OAEs are often unrecordable at this age because of amniotic fluid and other debris in the ear canal. ${ }^{19}$

The role of universal neonatal screening in the UK is even more uncertain because all children are already screened in infancy, albeit at around 7 months of age. A controlled trial of OAE screening is therefore being conducted in Wessex. The main aims are to determine the potential yield and resource implications of universal screening in this country.

There are only a few reports of OAE screening beyond the neonatal period, ${ }^{22-24}$ although several groups of children have been identified in which it may be useful. These include children with learning difficulties and those who are at risk of sensorineural hearing loss after meningitis or the use of ototoxic drugs. ${ }^{72-26}$ Research in children has probably been hampered by reports of difficulties specific to this age group. Some investigators have found that older infants and toddlers simply will not tolerate the test procedure. ${ }^{1224}$ Others have found that OAEs can be recorded with a little patience and distraction. ${ }^{23} 27$

The major problem with OAE screening is its lack of specificity. In full term infants and young children false positives are largely due to contamination of the OAE response by other sounds. ${ }^{23} 2728$ Contaminating noise can be both external (in the test environment) and internal (from the child). Internal noise, from processes such as breathing and swallowing, is particularly troublesome.

Linked to the problem of noise is the issue of OAE analysis and pass/fail criteria. In ideal conditions, for example a cooperative patient in a soundproof room, OAEs are clearly either present or absent. However, in the real world of paediatric audiology it is not always possible to distinguish the sounds of an OAE from those of background noise. It is here that simple and efficient pass/fail criteria are needed. Most OAE criteria are derived from analyses of the reproducibility and/or signal-to-noise ratio of the whole OAE waveform. They are vulnerable to contamination by noise at certain frequencies and so they frequently fail normal ears. This problem can be partially alleviated by limiting the analysis to a few, selected frequencies. ${ }^{23} 2728$ Screening criteria based on this approach appear to improve specificity without any loss of sensitivity.

\section{Otoacoustic emissions in audiological diagnosis}

OAEs have other uses besides screening. Emissions are produced by the cochlear amplifier, which is believed to function without any afferent input to the central nervous system. ${ }^{6}$ The absence or presence of OAEs can therefore be used to distinguish between the sensory and neural components of sensorineural deafness. This approach can be used for individual assessment, for example in determining whether a child with known sensorineural deafness will benefit from a cochlear implant. It can also be useful in research. We are currently using a combination of OAEs and ABRs to determine the precise site of the lesion in postmeningitic hearing loss.

Two points should be made about OAEs in sensori- 
neural deafness. The first is that, because OAEs are generally present in patients with a retrocochlear (neural) hearing loss, any such patients would pass an OAE screen. Fortunately, such hearing losses are vanishingly rare in infancy. This phenomenon is therefore unlikely to cause many false negatives in neonatal screening. ${ }^{29}$ The second point is that patients with acoustic neuromas often do not produce OAEs. ${ }^{70}$ This unexpected finding is probably due to the neuroma causing a coincident sensory hearing loss secondary to cochlear ischaemia. ${ }^{30}$ OAEs are therefore unreliable in diagnosing this condition.

Because OAEs travel through the middle ear, they are also affected by middle ear disease. Children with glue ear do not produce emissions. ${ }^{723}$ This means that OAEs cannot differentiate between cochlear and conductive deafness. Accordingly, OAEs should always be used in conjunction with tympanometry. In fact, it is likely that combined OAE/tympanographs will shortly be developed. ${ }^{27}$

\section{Future developments}

From the discussion of OAEs in screening, it is clear that the test can be improved by strategies that diminish the influence of noise. The number of false positive results can be reduced by adopting limited frequency screening, 232728 but other approaches are also being explored. The signalto-noise ratio may be improved by using stimuli that evoke a stronger response. ${ }^{31}$ The ratio can also be increased by recording a greater number of responses. Although this strategy seems undesirable, the introduction of faster $\mathrm{OAE}$ devices $^{32}$ should make it more practical in the future.

To date, most OAE research in children has concentrated on transient evoked emissions. It is likely that another type of $\mathrm{OAE}$, the distortion product, will receive increasing attention. Distortion products are particularly appealing because, unlike transient emissions, they can be used to produce displays that closely match the patient's audiogram. ${ }^{83}$ They may also be more sensitive to high frequency hearing loss. ${ }^{33}$ Furthermore, despite requiring more complex equipment than transient emissions, distortion products may be quicker to record. ${ }^{34}$

With these and other developments, which may include automated analysis, it is certain that OAE devices will become increasingly available. They are sure to be introduced into more paediatric audiology clinics. If there is any doubt about a child's hearing status, a quick and objective test could then be performed without the need for tertiary referral. This will also reduce the requirement for measurement of ABRs, a technique that often requires sedation, or even anaesthesia, in young children. ${ }^{11}$ It is also possible that OAEs will be used for more screening purposes. In the UK, all children have hearing tests at 6 to 8 months and at school entry. Eventually OAEs could augment, or even replace, these screening procedures.

\section{Conclusions}

OAEs are an extremely sensitive method of detecting hearing impairment. Because of their objectivity and ease of measurement, they are of particular use in neonatal screening. In time, they may also be used for screening older children. Another use of OAEs is as part of a battery of tests in audiological diagnosis.

It is important, however, to recognise the limitations of isolated OAE recordings. The presence of an OAE shows that the sensory components of the cochlea are functioning. The patient could still have a central hearing loss. Absent or unidentifiable OAEs are caused by cochlear and conductive hearing losses, but also by contaminating noise. In other words: if you can record an OAE, the inner ear is working; if you can't, it doesn't necessarily mean it isn't.

Bath Unit for Research into Paediatrics, MARTIN RICHARDSON

Royal United Hospital,

Bath BA1 3NG

1 von Békésy G. Experiments in hearing. New York: Chapman and Hall, 1960.

2 Sellick PM, Patuzzi R, Johnstone BM. Measurement of basilar membrane motion in the guinea pig using the Mossbauer technique. $\mathcal{F}$ Acoust Soc Am 1982; 72: 131-41.

$3 \mathrm{Kemp}$ DT. Stimulated acoustic emissions from within the human auditory system. F Acoust Soc Am 1978; 64: 1386-91.

4 Wilson JP. Theory of tinnitus generation. In: Hazell JWP, ed. Tinnitus. Edinburgh: Churchill Livingstone, 1987: 20-45.

5 Davis H. An active process in cochlear mechanics. Hear Res 1983; 9: 79-80. 6 Ashmore JF. The cellular machinery of the cochlea. Exp Physiol 1994; 79: 113-34.

7 Kemp DT, Bray P, Alexander L, Brown AM. Acoustic emission cochleography - practical aspects. Scand Audiol 1986; 15 (suppl 25): 71-95.

8 Probst R, Lonsbury-Martin BL, Martin GK. A review of otoacoustic emissions. F Acoust Soc Am 1991; 89: 2027-67.

9 Kemp DT, Ryan S, Bray P. A guide to the effective use of otoacoustic emissions. Ear Hear 1990; 11: 93-105.

10 Alberti PW, Hyde ML, Corbin H, Riko K, Abramovich S. An evaluation of BERA for hearing screening in high-risk neonates. Laryngoscope 1983; 93: BERA for

11 Mason S, McCormick B, Wood S. Auditory brainstem response in paediatric audiology. Arch Dis Child 1988; 63: 465-7.

12 Stevens JC, Webb HD, Hutchinson J, Connell J, Smith MF, Buffin JT. Click evoked otoacoustic emissions compared with brain stem electric response. Arch Dis Child 1989; 64: 1105-11.

13 Kennedy CR, Kimm L, Cafarelli Dees D, et al. Otoacoustic emissions and auditory brainstem responses in the newborn. Arch Dis Child 1991; 66: $1124-9$.

14 Meredith R, Stephens D, Hogan S, Cartlidge PHT, Drayton M. Screening for hearing loss in an at-risk neonatal population using evoked otoacoustic emissions. Scand Audiol 1994; 23: 187-93.

15 Watkin PM, Baldwin M, McEnery G. Neonatal at risk screening and the identification of deafness. Arch Dis Child 1991; 66: 1130-5.

16 Copman J. Deafness: ever heard of it? Delayed recognition of permanent hearing loss. Pediatrics 1987; 79: 206-13.

17 Davis A, Wood S. The epidemiology of childhood hearing impairment: factors relevant to planning of services. Br 7 Audiol 1992; 26: 77-90.

18 Ramkalawan TW, Davis AC. The effects of hearing loss and age of intervention on some language metrics in young hearing-impaired intervention on some language metrics

19 Maxon AB, White KR, Vohr BR, Behrens TR. Using transient evoked otoacoustic emissions for neonatal hearing screening. Br $\mathcal{f}$ Audiol 1993; 27: 149-53.

20 National Institutes of Health. Early identification of hearing impairment in infants and young children. Bethesda: National Institutes of Health, 1993.

21 Bess FH, Paradise JL. Universal screening for infant hearing impairment not simple, not risk-free, not necessarily beneficial, and not presently justified. Pediatrics 1994; 93: 330-4.

22 Nozza RJ, Sabo DL. Transiently evoked OAE for screening school-age children. Hearing fournal 1992; 45: 29-31.

23 Richardson MP, Williamson TJ, Lenton SW, Tarlow MJ, Rudd PT. Otoacoustic emissions as a screening test for hearing impairment in children. Arch Dis Child 1995; 72: 294-7.

24 Fortnum H, Farnsworth A, Davis A. The feasibility of evoked otoacoustic emissions as an in-patient hearing check after meningitis. $\mathrm{Br} \mathcal{F}$ Audiol 1993; 27: 227-31.

25 Baldwin M, Watkin P. The clinical application of otoacoustic emissions in paediatric audiological assessment. F Laryngol Otol 1992; 106: 301-6.

26 Norton SJ, Widen JE. Evoked otoacoustic emissions in normal-hearing

infants and children: emerging data and issues. Ear Hear 1990; 11: 121-7.
Bray P, Kemp D. An advanced cochlear echo technique suitable for infant screening. Br f Audiol 1987; 21: 191-204.

28 Brass D, Kemp DT. The objective assessment of transient evoked otoacoustic emissions in neonates. Ear Hear 1994; 15: 371-7.

29 Lutman ME. Reliable identification of click-evoked otoacoustic emission using signal-processing techniques. Br $\mathcal{F}$ Audiol 1993; 27: 103-8.

30 Cane MA, Lutman ME, O'Donoghue GM. Transiently evoked otoacoustic emissions in patients with cerebellopontine angle tumors. Am $\mathcal{F}$ Otol 1994 15: $207-16$.

31 Neumann J, Uppenkamp S, Kollmeier B. Chirp evoked otoacoustic emissions. Hear Res 1994; 79: 17-25.

32 Thornton ARD. High rate otoacoustic emissions. F Acoust Soc Am 1993; 94: 132-6.

33 Lonsbury-Martin BL, McCoy MJ, Whitehead ML, Martin GK. Clinical testing of distortion-product otoacoustic emissions. Ear Hear 1993; 14: 11-22.

34 Brass D, Kemp DT. Quantitative assessment of methods for the detection of otoacoustic emissions. Ear Hear 1994; 15: 378-89. 